\author{
Military Technical College \\ Kobry El-Kobbah, \\ Cairo, Egypt
}

\author{
$8^{\text {th }}$ International Conference \\ on Electrical Engineering \\ ICEENG 2012
}

\title{
Narrowband Interference Mitigation in IR-UWB Communication Systems Using Code Sequence Selection
}

\author{
By \\ Ehab M. Shaheen *
}

\section{Abstract:}

In this paper we will show that the impact of narrowband interference signal on the performance of direct sequence ultra wide band communication system in a Log-normal flat fading channel can be easily mitigated. The idea of the proposed mitigation technique is based on adapting the ultra wideband transmitted spectrum with the suitable selection of the direct sequence pseudo random code sequence in order to counteract narrowband interference and at the same time to guarantee low spectral emissions over existing narrowband communication systems.

\section{Keywords:}

Ultra-wideband, Narrow band interference, Log-normal fading channel; code adaptation.

* Egyptian Armed Forces

\section{Introduction:}


In recent years, the impulse radio ultra-wideband (IR-UWB) specially the Direct sequence (DS) UWB system has been proposed as a candidate technology at the physical layer of the high-speed short-range wireless personal area networks (WPAN). Since IR-UWB systems are planned to coexist with other legacy narrowband systems, the transmission power of the UWB devices is strictly limited by the Federal Communications Commission (FCC) agency so that the pre-existing narrowband systems will be affected by the UWB signals only at a negligible level. However, these narrowband systems may cause severe interference to the UWB system which may jam the UWB receiver completely [1]-[5].

In order to reduce the interference to existing narrowband systems, the FCC also imposed a power restriction on UWB communication systems, where the power spectral density levels are limited to $-41.3 \mathrm{dBm} / \mathrm{MHz}$. However, narrowband signals that exist in the UWB range may exhibit a high power spectral density (PSD) levels compared to the PSD of UWB signals as seen by a UWB receiver. As a result, one would expect a degradation of the UWB bit error rate (BER) performance. Therefore, the issue of the interference cancellation is crucial to the UWB systems.

Narrowband interference (NBI) suppression in UWB communication systems has been extensively studied before and it can be borrowed from those used in code division multiple access spread-spectrum systems [6-8]. In [9], the authors studied the use of a notch filter to suppress NBI for time hopping UWB systems. A narrowband suppression scheme based on minimum mean-square-error Rake reception was examined for UWB systems in [10]. In [11], the use of a non-linear prediction filter in DS UWB systems to reject NBI had been studied. However, these techniques are performed in UWB receivers to reduce the effect of NBI on UWB signals, while the interference caused by UWB devices to narrowband services must also be mitigated.

To this end, the main objective of this paper is to propose an approach which adopts the pseudo-random code sequence used in the IR-UWB system to mitigate the impact of NBI by shaping the UWB signal spectrum. With the convenient selection of such code sequence the impact of NBI signal can be mitigated or suppressed.

The paper is organized as follows. The system model is described in section 2. In section 3 the idea of the proposed approach is presented. Section 4 presents representative numerical results of system performance with and without the use of the proposed approach and validated with simulation. Finally, section 5 draws the conclusions. 


\section{System Model:}

\section{A. The Desired Signal}

The transmitted UWB signal can be written either in the form of a Time hopping pulse position modulation (TH-PPM) or in the form of a DS binary phase shift keying (BPSK) as

$$
\begin{aligned}
& S_{B P S K}^{D S}(t)=\sqrt{E_{b}} \sum_{i} d_{i} \sum_{j=-\infty}^{\infty} C_{j} p\left(t-j T_{f}-i T_{b}\right) \\
& S_{P P M}^{T H}(t)=\sqrt{E_{b}} \sum_{\mathbf{i}} \sum_{\mathbf{j}=-\infty}^{\infty} \mathbf{p}\left(\mathbf{t}-\mathbf{C}_{\mathbf{j}} \mathbf{T}_{\mathbf{c}}-\delta \mathbf{d}_{\left|\frac{\mathbf{i}}{N_{\mathbf{s}}}\right|}-\mathbf{j}_{\mathbf{f}}-\mathbf{i} \mathbf{T}_{\mathbf{b}}\right)
\end{aligned}
$$

where $p(t)$ is the shape of the transmitted pulse with pulse width Tm. In DS system $d_{i}$, is the transmitted $i^{\text {th }}$ binary data bit and composed of equally likely bits. $T_{f}$ is the frame duration, the bit duration can be represented as $T_{b}=N_{s} T_{f}$ with bit energy $E_{b} . N_{s}$ is the number of pulses transmitted per bit. $C_{j}$ in the DS bipolar code sequence, $C_{j} \quad\{-1,+1\}$. In TH-PPM system, $\mathrm{T}_{\mathrm{c}}$ is the TH chip width, $\mathrm{C}_{\mathrm{j}}$ is the TH code $\{0,1 ; \ldots ; \mathrm{Nh}-1\}$, such

that an additional time shift of $\mathrm{C}_{\mathrm{j}} \mathrm{T}_{\mathrm{c}}$ is introduced when the $\mathrm{j}^{\text {th }}$ pulse is transmitted. is the $\mathrm{i}^{\text {th }}$ binary data bit transmitted and composed of equally likely symbols (or bits). Finally, = is the modulation index (the time shift added to a pulse with an optimal value of $20 \%$ of a pulse width).

\section{B. The NBI Signal Model}

The considered NBI signal is the sum of " $\mathrm{N}_{\mathrm{i}}$ " tone interferers. The NBI signal can be written as

$$
I(t)=\sum_{n=1}^{N_{i}} \sqrt{\frac{2 I}{N_{i}}} \cos \left(2 \pi f_{n} t+\varphi_{n}\right)
$$

where " $\mathrm{f}_{\mathrm{n}}$ " is the $\mathrm{n}^{\text {th }}$ interference frequency, and " $\varphi_{\mathrm{n}}$ " are independent and identically distributed (i.i.d.) random phases due to modulation and i.i.d. symbols. I is the total transmitted power of the interference signal.

\section{Channel Models}


Due to the huge UWB bandwidth, the propagation channel for UWB signals is frequency-selective and it cannot be modeled as a flat fading channel. However, some wireless systems that use the UWB technology such as the wireless sensor networks are characterized by size and energy constraints. These constraints are imposed on each node that necessitate the use of simple devices. The use of a one finger Rake receiver can be considered as a sub-optimal solution for simple and low cost communication systems. In this case the channel can be modeled as a flat fading.

The impulse response of the UWB system in a flat fading channel is given by [12]

$$
h_{s}(t)=a_{s} \delta\left(t-\tau_{s}\right)
$$

where $a_{s}$ is the channel gain coefficient and $\tau_{s}$ is the channel time delay.

The channel impulse response for NBI signal can be written as

$$
h_{i}(t)=\alpha_{i} \delta\left(t-\tau_{i}\right)
$$

where $\alpha_{\mathrm{i}}$ is the Rayleigh distributed channel gain and $\tau_{\mathrm{i}}$ is the corresponding time delay. The received signal can be written as

$$
r(t)=S_{r}(t)+I_{r}(t)+n(t)
$$

where $n(t)$ is the additive white Gaussian noise (AWGN) with two sided power spectral density No/2.

The desired received signal for the DS-BPSK system, $\mathrm{S}_{\mathrm{r}}(\mathrm{t})=S_{B P S K}^{D S}(t) * h_{S}(t)$, where $*$ is the convolution integral, it can be written as

$$
S_{r}(t)=\sqrt{E_{b}} \sum_{i} d_{i} \sum_{j=-=}^{\infty} a_{s} C_{j} p\left(t-j T_{f}-i T_{b}-\tau_{s}\right)
$$

For a TH-PPM system, it can be written as

$$
S_{r}(t)=\sqrt{E_{b}} \sum_{i} \sum_{j=-\infty}^{\infty} a_{s} p\left(t-C_{j} T_{c}-\delta d_{\left|\frac{i}{N_{s}}\right|}-j T_{f}-i T_{b}-\tau_{s}\right)
$$

The interference term, $\mathrm{I}_{\mathrm{r}}(\mathrm{t})=I(t) * \boldsymbol{h}_{i}(t)$, it can be written as 
$I_{r}(t)=\sqrt{\frac{2 I}{N_{i}}} \sum_{n=1}^{N_{i}} \alpha_{i} \cos \left(2 \pi f_{n}\left(t-\tau_{i}\right)+\varphi_{n}\right)$

Without loss of generality, it is assumed that the UWB signal channel impulse response, $\mathrm{h}_{\mathrm{s}}(\mathrm{t})$, and the interferer channel impulse response, $\mathrm{h}_{\mathrm{i}}(\mathrm{t})$, are normalized so that $E\left\{a_{S}^{2}\right\}=$ $E\left\{\alpha_{i}^{2}\right\}=1$, where $\mathrm{E}[$.] denotes the expectation operator.

\section{Interference Analysis}

In this section, the possible coexistence between a UWB communication system and narrowband system operating within the same frequency band is investigated. In figure 1 , a sketch of the scenario inside the critical area identified, is depicted. In particular, the figure highlights such coexistence problem. In this scenario, we have to determine first the impact of NBI on the UWB system and then adapt the UWB spectrum to guarantee a possible coexistence.

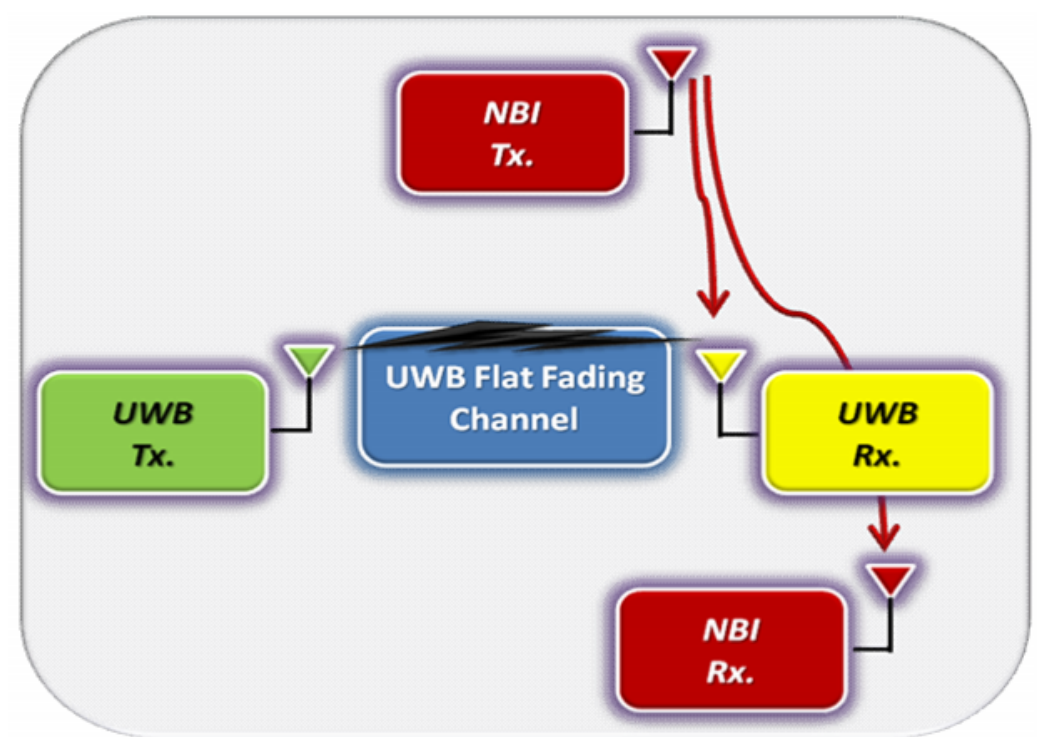

Fig. 1 The coexisting scenario between a UWB communication system and a narrowband interference Signal.

The UWB correlation mask can be written as

$\bar{m}(t)=\sum_{l=0}^{L} a_{l} \cdot m(t)$

where $\mathrm{m}(\mathrm{t})$ for a TH-PPM system can be written as 
$m(t)=p\left(t-j T_{f}-C_{j} T_{c}-\tau_{s}\right)-p\left(t-j T_{f}-C_{j} T_{c}-\tau_{s}-\delta\right)$

and for a DS-BPSK system, it can be written as

$m(t)=C_{j} p\left(t-j T_{f}-\tau_{s}\right)$

the matched filter receiver, which can be written for a DS-UWB signal as

$|M(f)|=2|P(f)|\left|\sum_{k=0}^{N_{S^{-1}}} C_{k} \cdot \exp \left(j 2 \pi f k T_{f}\right)\right|$

whereas for a TH-PPM system, the transfer function $|\mathrm{M}(\mathrm{f})|$ is given by

Where $\mathrm{P}(\mathrm{f})$ is the Fourier Transform of the six derivative Gaussian UWB pulse $\mathrm{p}(\mathrm{t})$ which can be written as

$P(f)=\frac{8 \pi^{3}}{3 \sqrt{1155 N_{s}}} \cdot \tau_{p}^{\frac{13}{2}} \cdot f^{6} \cdot \exp \left(-\frac{\pi f^{2} \tau_{p}^{2}}{2}\right)$

The performance of IR- UWB system in the presence of NBI in a scenario characterized by Log-normal flat fading on the UWB link had been presented in [13]. To this aim, following the approach developed in [13], the bit error probability (BEP) of the IR-UWB communication system in the presence of $\mathrm{N}$ tone interferers can be written as [13]

$P_{e}=\frac{1}{\sqrt{\pi}} \sum_{i=1}^{N} \omega_{i}\left[Q\left(\sqrt{\gamma \exp \left(2 \sqrt{2} b_{i} \sigma_{r}+2 \mu_{r}\right)}\right)\right]$ 
where $\omega_{i}$ and $b_{i}$ are the weights and the associated roots of the Hermite polynomial respectively where $\omega_{i}$ and $b_{i}$ are found in [14]. $\mathrm{N}$ are the number of samples points to use for this approximation.

$\mu_{r}$ and $\sigma_{r}$ are the mean and standard variation of the Log-normal random variable, $\mathrm{a}_{\mathrm{s}}$. Finally, $\gamma$ is the signal to interference plus noise power ratio (SINR), which can be written for a DS-BPSK UWB system as [13]

$\gamma_{D S}=\left[\left(\frac{2 E_{b}}{N_{o}}\right)^{-1}+\left(\frac{4 \cdot S I R \cdot N_{i} \cdot T_{b}}{\Sigma_{n}^{N_{i}}\left|M\left(f_{n}\right)\right|^{2}}\right)^{-1}\right]^{-1}$

$\gamma_{T H}=\left[\left(\frac{E_{b}(1-\beta)}{N_{o}}\right)^{-1}+\left(\frac{\operatorname{SIR} \cdot N_{i} \cdot T_{b} \cdot(1-\beta)^{2}}{\Sigma_{n}^{N_{i}}\left|M\left(f_{n}\right)\right|^{2}}\right)^{-1}\right]^{-1}$

Where SIR is the signal to interference power ratio, and $M\left(f_{n}\right)$ is the transfer function of the matched filter. $\beta$ is the is the correlation coefficient between the two UWB pulses, $p(t)$ and $\mathrm{p}(\mathrm{t}-\delta)$ for the two bits $(0,1)$ respectively. $\beta$ can be defined as

$\beta=\int_{-\infty}^{\infty} p(t) p(t-\delta) d t$

It can be seen that $\mathrm{M}(\mathrm{f})$ as presented in equations (13) and (14) and consequently the BEP expression presented in equations (17) depend on the sequence $\left\{C_{j}\right\}$, thus by suitable adaptation of the code sequence $\left\{\mathrm{C}_{\mathrm{j}}\right\}$, a null can be introduced at the exact narrowband system operating frequencies.

Assuming independent and equi-probable bits, $\mathrm{d}_{\mathrm{j}}$, the PSD of the IR-UWB transmitted signals (1) and (2) has the same shape of the matched filter frequency except for an irrelevant constant. Thus, the UWB system can potentially detect the NBI operating frequency and select the best spreading sequence that minimizes the impact of interference at the receiver. By doing this, at the same time, the transmitted spectrum will have a minimum level around $f_{i}$, which in turn will guarantee that the mutual interference remains below a certain level, allowing coexistence and better usage of the spectrum.

\section{Simulation and Numerical Results}

In this section, the considered DS-BPSK system with six derivative Gaussian received pulse has a pulse duration $\tau_{\mathrm{p}}=0.192 \mathrm{~ns}$, a frame length $\mathrm{T}_{\mathrm{f}}=100 \mathrm{~ns}$. The two 
possible spreading sequences: $\left\{\mathrm{C}_{1}\right\}=\{-1,-1,+1,-1,-1,+1\}$ and $\left\{\mathrm{C}_{2}\right\}=\{+1,+1,-1$, $-1,-1,+1\}$ of length $\mathrm{N}_{\mathrm{s}}=6$. The NBI signal is modeled as a single tone signal operating at $5.742 \mathrm{GHz}$.

Figure (2) depicts the normalized matched filter transfer function in a frequency range around the NBI operating frequency for two possible code sequences. It can be seen that it is better to adapt the first code sequence than the second one as the latter gives higher level of interference at the output of the matched filter receiver.

Figure (3) depicts the effect of adapting the suitable code sequence which guarantees the lower interference at the matched filter receiver output on the BER performance of a DS-UWB communication system in the presence of a single tone NBI signal operating at $5.742 \mathrm{GHz}$ in a Log-normal flat fading channel with $\mathrm{dB}$-spread value $=2 \mathrm{~dB}$. It can be seen that with the adaptation of code 1 outperforms the adaptation of code 2 .

Figure (4) depicts the effect of adapting the suitable code sequence which guarantees the lower interference at the matched filter receiver output on the BER performance of a DS-UWB system in the presence of a single tone NBI signal operating at $5.74 \mathrm{GHz}$ in a Log-normal flat fading channel and validated with simulation.

It can be seen that the DS-UWB system with the adaptation of code 2 outperforms the adaptation of code 1 and the impact of the NBI signal is completely removed.

For a TH-PPM system with a six derivative Gaussian received pulse has a pulse duration $\tau_{\mathrm{p}}=0.5 \mathrm{~ns}$, a frame length $\mathrm{T}_{\mathrm{f}}=100 \mathrm{~ns}, \beta=-0.824$ and modulation index $\delta=$ $0.3 \mathrm{~ns}$. The two possible spreading sequences for $\mathrm{N}_{\mathrm{s}}=4$ are $: \mathrm{C}_{1}=\{0,1,5,20\}$ and $\mathrm{C}_{2}=$ $\{1,5,7,15\}$. 


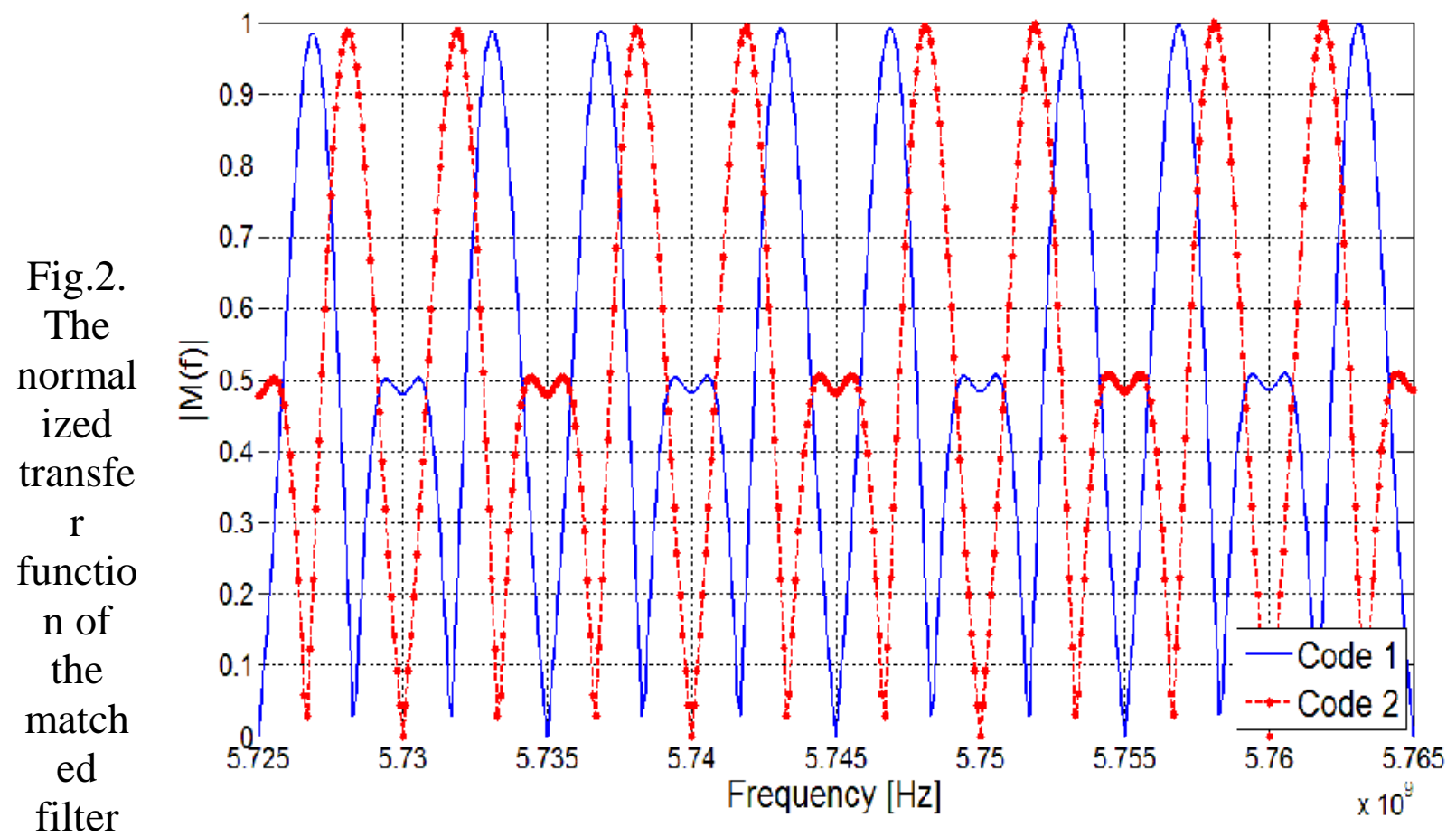

filter

receiver for two different code sequences for DS-BPSK system.

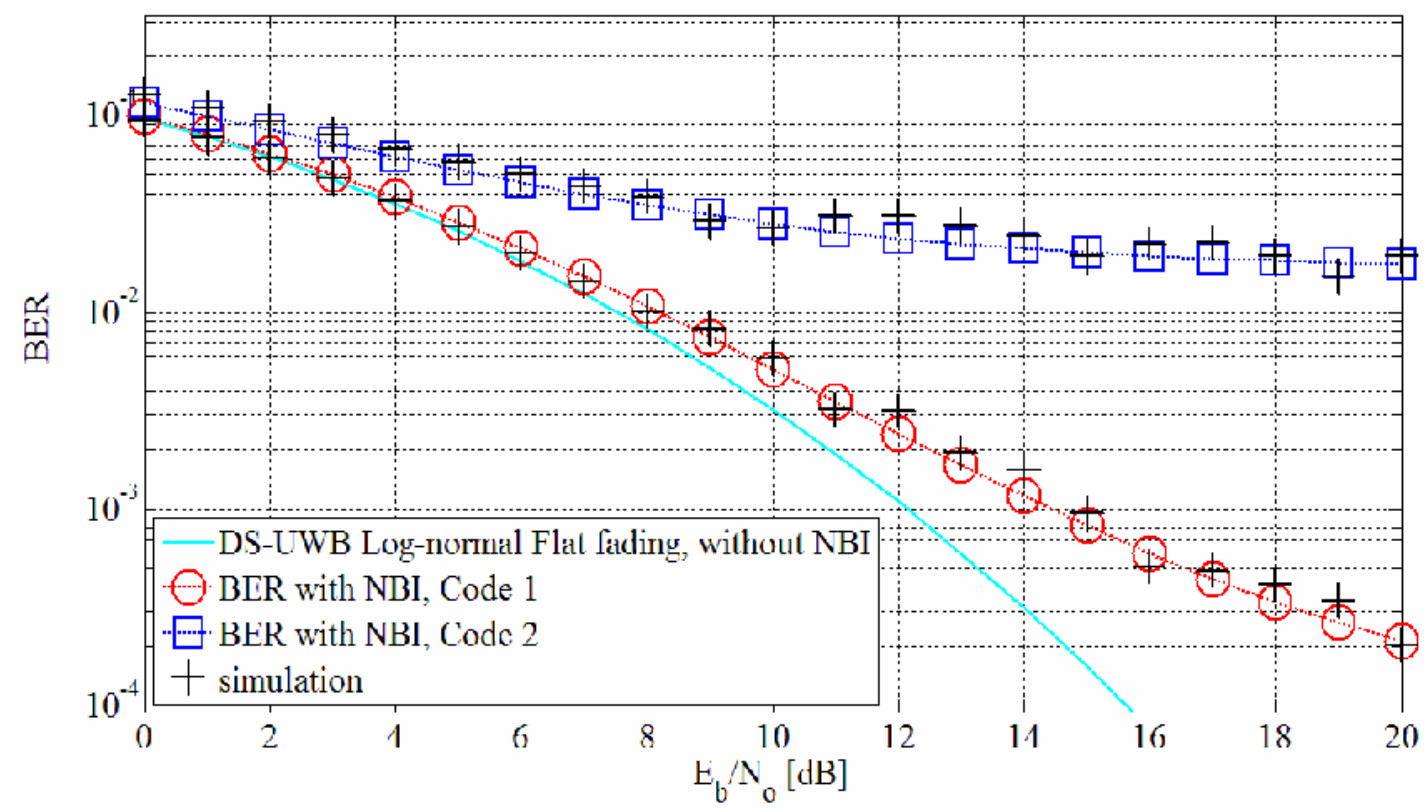

Fig.3. BER performance of a DS-BPSK UWB system in the presence of NBI, $\mathrm{SIR}=-25 \mathrm{~dB}$ with two different code sequences, $\mathrm{dB}$-spread $=2 \mathrm{~dB}, \mathrm{f}_{\mathrm{i}}=5.742 \mathrm{GHz}$. 


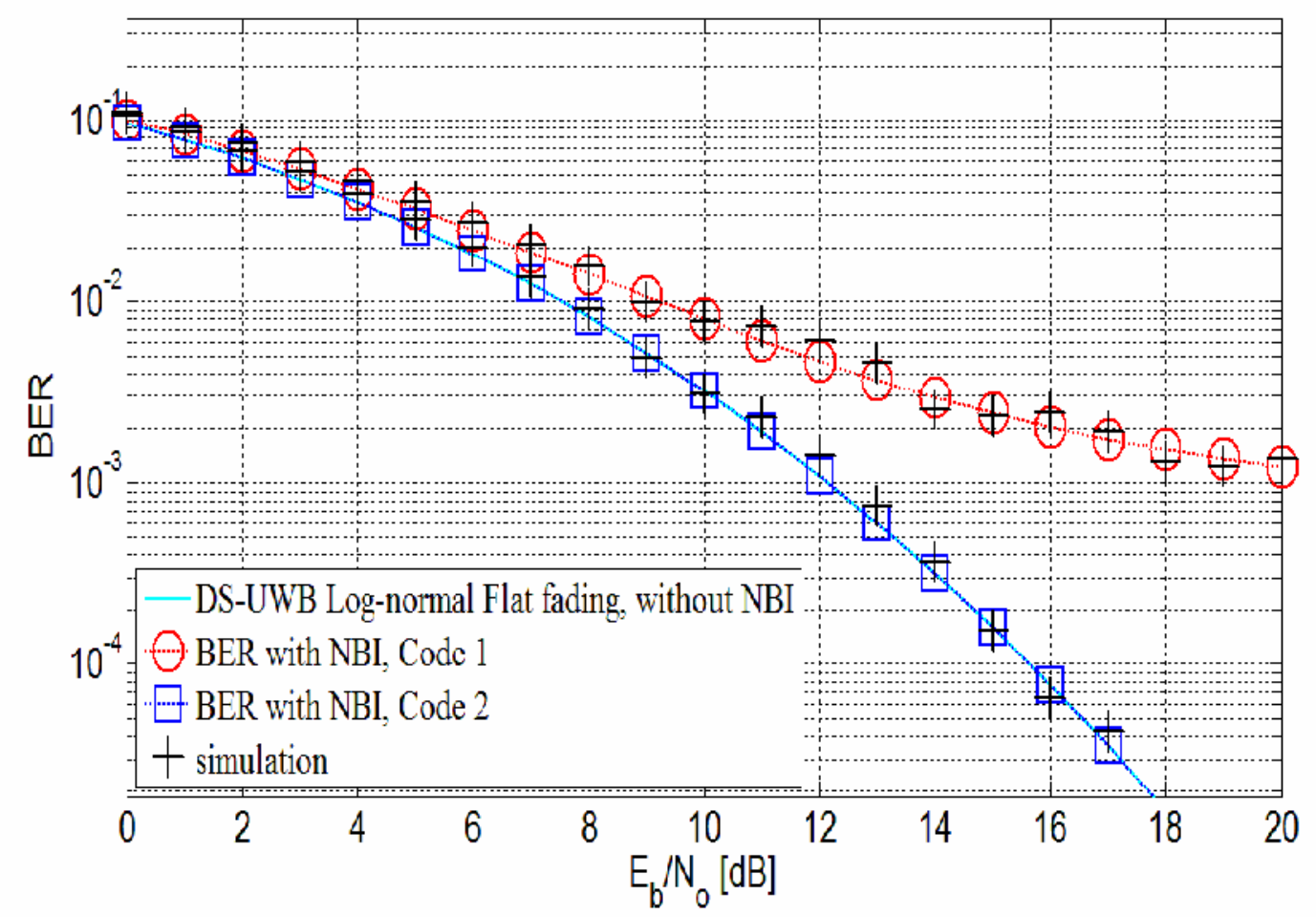

Fig.4. BER performance of a DS-BPSK UWB system in the presence of NBI, $\mathrm{SIR}=-25 \mathrm{~dB}$ with two different code sequences, $\mathrm{dB}$-spread $=2 \mathrm{~dB}, \mathrm{f}_{\mathrm{i}}=5.74 \mathrm{GHz}$.

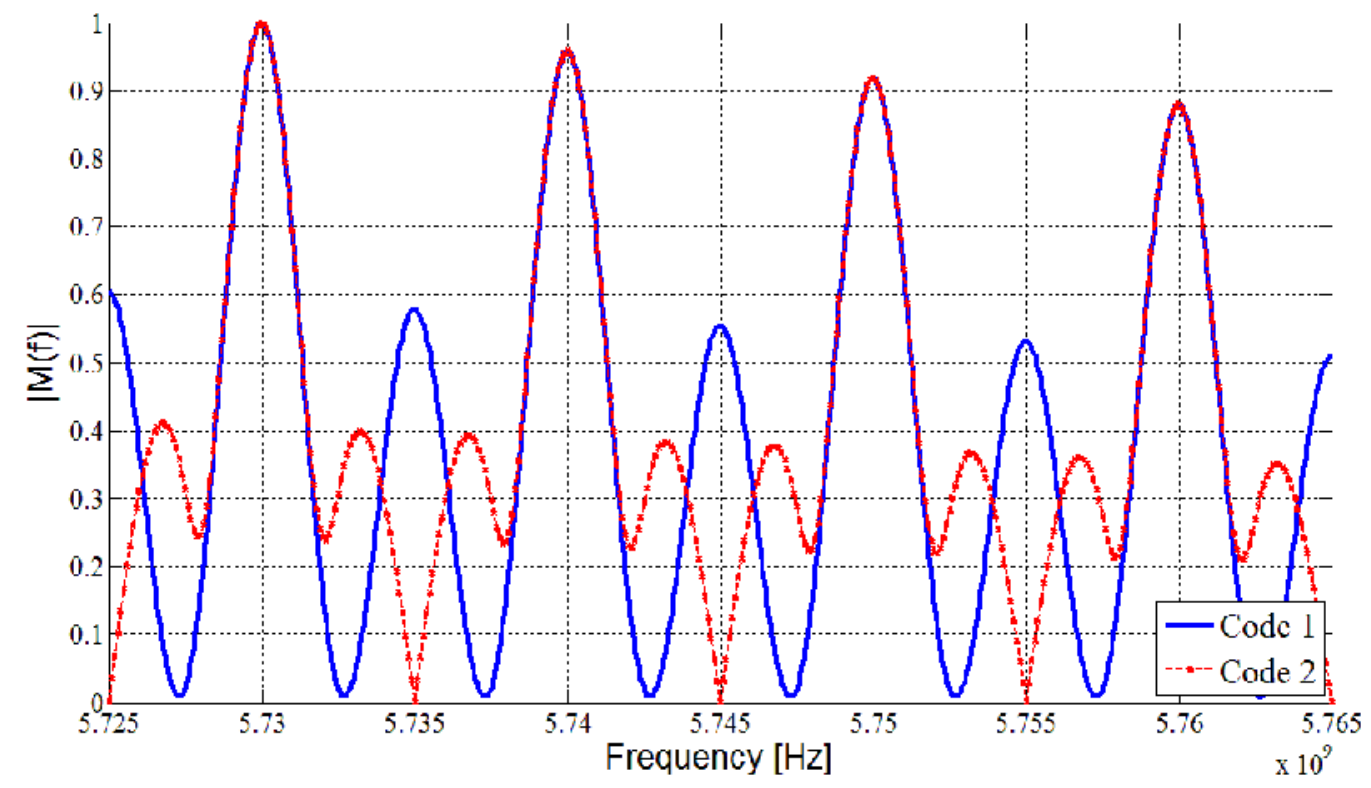

Fig.5. The normalized transfer function of the matched filter receiver for two different code sequences for TH-PPM system.

Figure (5) depicts the normalized matched filter transfer function in a frequency range 
around the NBI operating frequencies for the two previously depicted possible code sequences.

Figure (6) depicts the effect of adapting the suitable code sequence (code 1) which guarantees the lower interference at the matched filter receiver output on the BER performance of a TH-PPM communication system in the presence of a single tone NBI signal operating at $5.75 \mathrm{GHz}$ and validated with simulation as depicted in figure (7). It can be seen that the TH-PPM system with the adaptation of code 1 outperforms the adaptation of code 2 and the impact of the NBI signal is completely removed.

\section{Conclusions}

The goal of this paper was to show the importance of suitably adapting the pseudo random code sequence used in the DS-UWB communication system in a Log-normal flat fading channel. As with the good choice of this code sequence a lower level of interference from the NBI signal on the performance of DS-UWB communication system can be guaranteed or it can be completely filtered out by the transfer function of

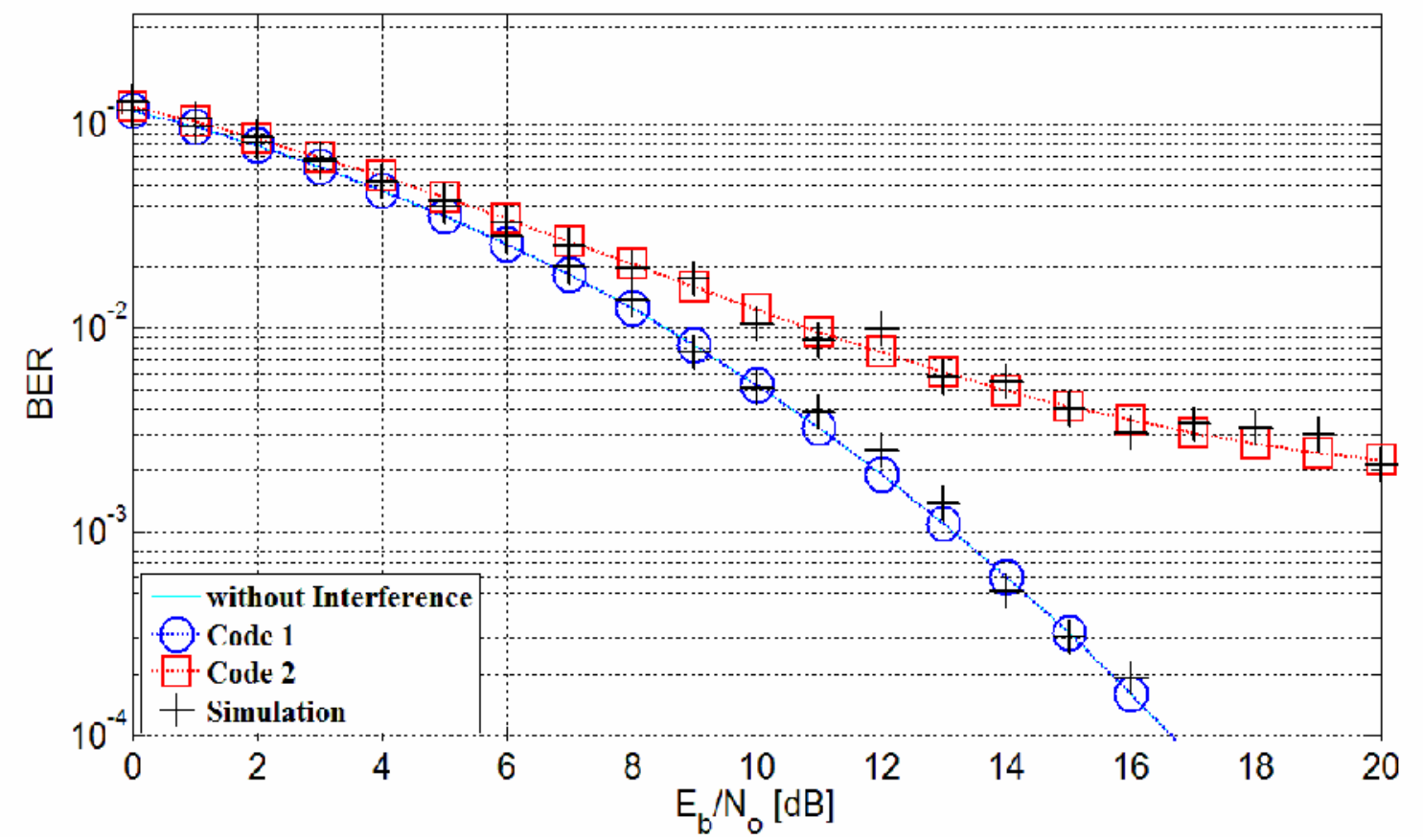

the

match

ed

filter

receiv

er.

The

analyt

result

$\mathrm{s}$ are

valida

ted

with

aid of

simul

ation. 
Fig.6. BER performance of a TH-PPM UWB system in the presence of NBI, $\mathrm{SIR}=-25 \mathrm{~dB}$ with two different code sequences, $\mathrm{dB}$-spread $=2 \mathrm{~dB}, \mathrm{f}_{\mathrm{i}}=5.75 \mathrm{GHz}$.

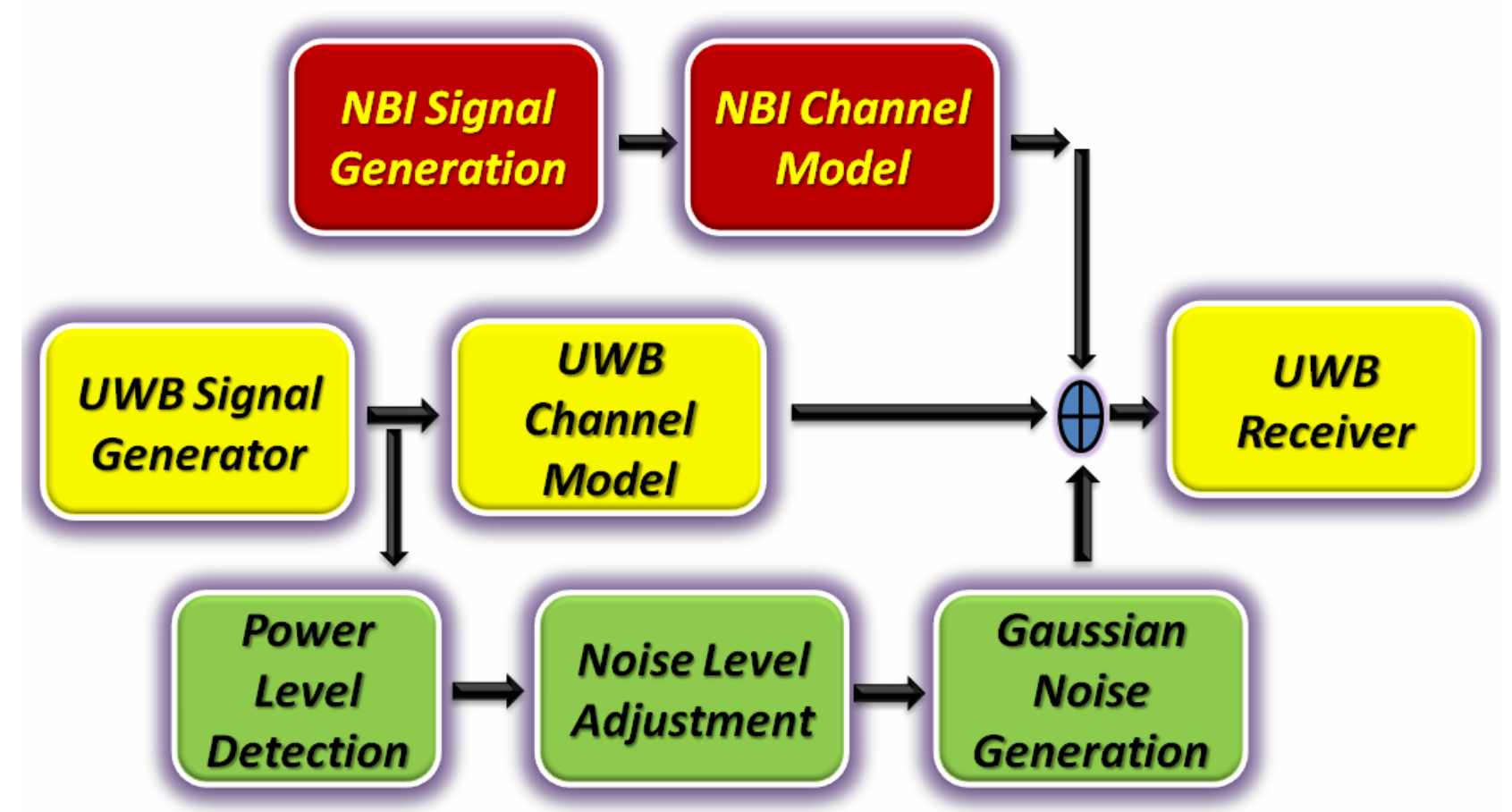

Fig.7. Simulation Block Diagram of the UWB System in the Presence of NBI.

\section{References:}

[1] B. Gaffney and A. D. Fagan, "Adaptive nonlinear narrow band interference rejection in ternary DS-UWB," in Proc. IEEE ICC, 2006, pp. 3146-3150. 
[2] I. Bergel, E. Fishler, and H. Messer, "Narrowband interference mitigation in impulse radio," IEEE Trans. Commun., vol. 53, no. 8, pp. 1278-1282, Aug. 2005.

[3] X. Chu and R. D. Murch, "The effect of NBI on UWB time-hopping systems," IEEE Trans.Wireless Commun., vol. 3, no. 5, pp. 1431-1436, Sep. 2004.

[4] Ehab M. Shaheen and M. El-Tanany, "BER analysis of UWB systems in the presence of narrowband interference in Log-normal multi-path fading channels," IEEE Military International Conference, Milcom2009, pp. 17, 18-21 October 2009.

[5] Ehab M. Shaheen and M. El-Tanany, "The impact of narrowband interference on the performance of UWB systems in the IEEE802.15.3a channel models," IEEE $23^{\text {rd }}$ Annual Canadian Conference on Electrical and Computer Engineering, CCECE2010, 2-5 May 2010.

[6] L. Milstein, "Interference rejection techniques in spread spectrum communications," Proc. IEEE, vol. 76, pp. 657-671, June 1988.

[7] L. Rusch and H. V. Poor, "Narrowband interference suppression in CDMA spread spectrum communications," IEEE Trans. Commun., vol. 42, pp. 1969-1979, Feb./Mar./Apr. 1994.

[8] H. V. Poor and X. Wang, "Code-aided interference suppression for DS/CDMA communications-I: interference suppression capability," IEEE Trans. Commun., vol. 45, pp. 1101-1111, Sep. 1997.

[9] J. Wang and W. T. Tung, "Narrowband interference suppression in time-hopping impulse radio ultra-wideband communications," IEEE Trans. Commun., vol. 54, no. 6, pp. 1057-1067, Jun. 2006.

[10] I. Bergel, E. Fishler, and H. Messer, "Narrowband interference suppression in timehopping impulse-radio systems," in Proc. IEEE Conf. Ultra Wide-band Syst. Technol., 2002, pp. 303-307.

[11] B. Gaffney and A. Fagan, "Adaptive nonlinear narrow band interference rejection in ternary DS-UWB," in Proc. IEEE Int. Conf. Commun., 2006, pp. 3146-3150.

[12] M. G. D. Benedetto, UWB Communication Systems: A Comprehensive Overview. Hindawi Publishing Corporation, 2006.

[13] Ehab M. Shaheen and M. El-Tanany, "Narrowband interference impact on the performance of UWB communication systems in Log-normal flat fading channels," IEEE $71^{\text {st }}$ Vehicular Technology Conference, VTC2010, 16-19 May 2010.

[14] M. Abramowitz and I. Stegun, Handbook of mathematical functions with formulas, graph, and mathematical tables. Dover, 9ed., 1971. 